\title{
Could hyponatremia be a marker of anastomotic leakage after colorectal surgery? A single center analysis of 1,106 patients over 5 years
}

\author{
S. A. Käser • U. Nitsche • M. Maak • C. W. Michalski • \\ C. Späth • T. C. Müller • C. A. Maurer • K. P. Janssen • \\ J. Kleeff • H. Friess • F. G. Bader
}

Received: 21 October 2013 / Accepted: 6 May 2014 / Published online: 23 May 2014

(C) Springer-Verlag Berlin Heidelberg 2014

\begin{abstract}
Purpose The aim of this study is to define the significance of hyponatremia as a marker of anastomotic leakage after colorectal surgery.

Methods All anastomoses in colorectal surgery performed at a single institution between July 2007 and July $2012(n=1,106)$ were retrospectively identified. Serum sodium levels and leukocyte values measured when an anastomotic leak was diagnosed by CT scan and/or surgical reintervention $(n=81)$ were compared to the values preferably on postoperative day 5 in the absence of an anastomotic leak $(n=1,025)$.

Results The leak rate in anastomoses of the rectum was $9.0 \%$, while the leak rate of the other anastomoses was $5.4 \%$. Mean serum sodium level was $138.8 \mathrm{mmol} / \mathrm{l}$ in the group with an anastomotic leak and $140.5 \mathrm{mmol} / 1$ in the group without. Hyponatremia $(<136 \mathrm{mmol} / \mathrm{l})$ was present in $23 \%$ of patients in the group with an anastomotic leak and in $15 \%$ in the group without $(p<0.001)$. In multivariate analysis, leukocytes and
\end{abstract}

This study has been presented at the meeting of the German Society of Coloproctology 2013 and at the meeting of the German Society of Surgery 2013.

Study conception and design was done by SAK, UN, MM, CWM, CAM, KPJ, JK, HF, and FGB; acquisition of data by SAK, UN, MM, CS, TCM, and FGB; analysis and interpretation of data by SAK, UN, MM, CWM, CS, TCM, CAM, KPJ, JK, HF, and FGB; drafting of manuscript by SAK, $\mathrm{UN}, \mathrm{CWM}, \mathrm{TCM}, \mathrm{JK}, \mathrm{HF}$, and FGB; and critical revision of manuscript by SAK, UN, MM, CWM, CS, TCM, CAM, KPJ, JK, HF, and FGB.

S. A. Käser · U. Nitsche $\cdot$ M. Maak · C. W. Michalski · C. Späth • T. C. Müller $\cdot$ K. P. Janssen $\cdot$ J. Kleeff $\cdot$ H. Friess $\cdot$ F. G. Bader Department of Surgery, Klinikum rechts der Isar, Technische Universität München, Ismaningerstrasse 22, 81675 Munich, Germany

S. A. Käser $(\square) \cdot$ C. A. Maurer

Department of Surgery, Cantonal Hospital of Baselland, Liestal, Rheinstrasse 26, 4410 Liestal, Switzerland

e-mail: sakaeser@swissonline.ch serum sodium level remained as significant markers of an anastomotic leak. As a marker of an anastomotic leak, hyponatremia had a specificity of $93 \%$ and a sensitivity of $23 \%$, while the presence of either leukocytosis or hyponatremia had a sensitivity of $68 \%$, a specificity of $75 \%$, a positive predictive value of $18 \%$, and a negative predictive value of $97 \%$.

Conclusions Hyponatremia could be a specific and relevant marker of anastomotic leakage after colorectal surgery. If hyponatremia and leukocytosis are present after colorectal surgery, anastomotic leakage should be suspected and a CT scan with rectal contrast dye is recommended.

Keywords Anastomotic leak · Inflammation markers · Sodium $\cdot$ Hyponatremia $\cdot$ Leukocytes

\section{Introduction}

Hyponatremia is the most frequent electrolyte disorder in clinical practice [1]. It is associated with a general increase of morbidity and mortality $[1,2]$. Mediated by osmotic release of vasopressin, hyponatremia typically occurs in patients with intravascular volume depletion as in fluid loss in to the third space [3]. However, recent research shows that direct immune neuroendocrine pathways connecting interleukin 6 to vasopressin seem to play an important role in the non-osmotic release of vasopressin in systemic inflammation $[4,5]$.

The relation between systemic inflammation and hyponatremia is well known from pneumonia [6] and in spontaneous bacterial peritonitis in liver cirrhosis [7]. Furthermore, a relation between hyponatremia and secondary peritonitis is known from case series in the 1960s [8]. Just recently, we showed that hyponatremia indeed was an indicator of peritonitis after perforation in infectious colonic disease [9]. While the specificity of hyponatremia for perforation in 
infectious colonic disease was $79 \%$, the sensitivity was as low as $31 \%$ [9]. Thus, the absence of hyponatremia could not exclude perforation in infectious colonic disease, while the presence of hyponatremia was a relevant marker of perforation in infectious colonic diseases [9].

Based on the abovementioned pathophysiological considerations and on the relation between hyponatremia and perforation in infectious colonic diseases [9], a relation between hyponatremia and anastomotic leakage after colorectal surgery was to be expected. We aimed to define the significance of hyponatremia and leukocytosis as predictors of anastomotic leakage after colorectal surgery.

\section{Materials and methods}

All surgery reports registered in a prospectively maintained database including all consecutive surgeries of the lower gastrointestinal tract performed at one institution (Klinikum rechts der Isar der Technischen Universität München) between July 2007 and July 2012 were analyzed in order to identify colonic or rectal anastomoses. In this database, diagnosis, type of surgery, presence of malignancy, comorbidities, and American Society of Anesthesiologists (ASA) score, as well as necessity of reoperation and follow-up data, were recorded. A chart review was performed to identify patients with an anastomotic leak. Diagnostic evaluation was only done in the case of suspicion of an anastomotic leak. It comprised CT scan with water-soluble contrast enema applied, conventional contrast enema, rectoscopy, and/or surgical reintervention in the case of obvious signs of anastomotic leakage such as fecal abdominal drainage. Abscesses in proximity of the anastomosis without extravasation of watersoluble contrast were accounted as a leakage.

Cutoff values for hyponatremia were not estimated; hyponatremia was already defined elsewhere as serum sodium levels $<136 \mathrm{mmol} / 1$ [10]. Leukocytosis was defined as leukocyte values $>10 \times 10^{9} / 1$. In patients without an anastomotic leak, the aim was to assess the serum sodium value and the leukocyte count preferably on day 5 after surgery, as on the one hand, the incidence of anastomotic leaks rises after day 5 postoperative [11], and on the other hand, routine blood test was rarely done after day 6 postoperative in these patients. As laboratory values were not measured daily, a ranking of preferred days of assessing the laboratory values (days 5, 4, 6, 7, 3, 8, and 2) was defined, in order to be as close as possible to day 5 postoperative. In patients with an anastomotic leak, the most recent laboratory results before the diagnosis was made were assessed.

Statistics Results are expressed as mean and standard deviation. Univariate analyses were done with Fisher's exact test (two-sided) for categorical data and with the Wilcoxon rank sum test for continuous variables. Multivariate analyses were done with logistic regression analysis. Significance was set at the $p<0.05$ level. The statistical analyses were performed with STATA 10 (StataCorp LP, Texas, USA).

\section{Results}

A total of 1,106 patients were identified in which an anastomosis was performed within the colon or the rectum between July 2007 and July 2012. Anastomotic leaks were diagnosed by (the sum does not equal $n=81$ ) the following: CT scan with watersoluble contrast $(n=52)$, conventional contrast enema $(n=4)$, rectoscopy $(n=8)$, and/or straightforward surgical reintervention in the case of obvious signs of anastomotic leakage $(n=24)$. An anastomotic leak after colorectal surgery occurred in 81 patients $(7.3 \%)$. The number of anastomotic leakages on each day after colorectal surgery is shown in Fig. 1.

The patient characteristics of the group of patients with and without an anastomotic leak are shown in Table 1 . The leak rate in rectal anastomoses $(n=588)$ was $9.0 \%$, while the leak rate of the other anastomoses $(n=518)$ was $5.4 \%$. The anastomotic leaks were managed as follows (the sum does not equal $n=81$ because the same patient could have several treatments): conservative treatment by antibiotics without intervention $(n=14)$, EndoVAC ${ }^{\circledR}$ therapy $(n=2)$, CT-guided pigtail placement and drainage of the abscess $(n=7)$, endoscopic closure of leak $(n=2)$, ileostomy $(n=27)$, correction or redo of the anastomosis $(n=22)$, discontinuity resection $(n=$ 23). One patient died before intervention could be done. The anastomotic leaks were graded after Rahbari [12] as follows: grade A $(n=14)$, grade $\mathrm{B}(n=8)$, and grade $\mathrm{C}(n=59)$. The overall 30-day mortality was $1.2 \%(n=1)$.

In patients without an anastomotic leak, the serum sodium level and the leukocyte count were assessed after mean

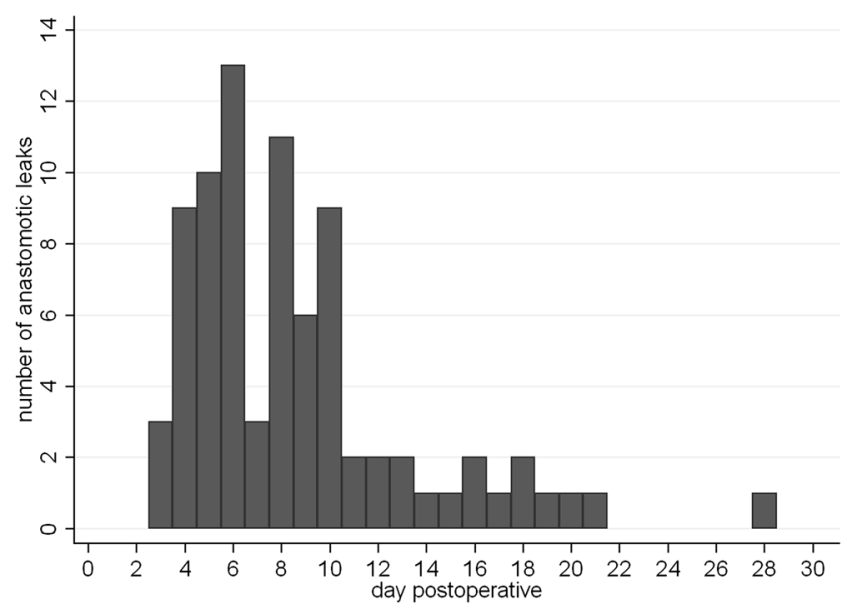

Fig. 1 The number of anastomotic leakages on each day after colorectal surgery 
Table 1 Characteristics of the analyzed patients with colonic or rectal anastomoses
ASA American Society of Anesthesiologists

${ }^{1}$ Two-sided Fisher's exact test

${ }^{2}$ Wilcoxon rank sum test

\begin{tabular}{llll}
\hline & $\begin{array}{l}\text { Anastomotic leak } \\
(n=81)\end{array}$ & $\begin{array}{l}\text { No anastomotic leak } \\
(n=1025)\end{array}$ & $p$ value \\
\hline $\begin{array}{l}\text { Mean age (range) } \\
\text { Male gender }\end{array}$ & 62.2 years (28-87) & $61.9(17-96)$ & $0.983^{2}$ \\
$\begin{array}{l}\text { Presence of hyponatremia } \\
\text { preoperative }\end{array}$ & $67 \%$ & $54 \%$ & $0.028^{1}$ \\
$\begin{array}{l}\text { Presence of ileus } \\
\text { Colorectal cancer }\end{array}$ & $2 \%$ & $9 \%$ & $0.081^{1}$ \\
Mean ASA score & $12 \%$ & $21 \%$ & $0.229^{1}$ \\
Presence of comorbidities & $41 \%$ & $43 \%$ & $0.727^{1}$ \\
Rectal cancer & 2.4 & 2.2 & $0.022^{2}$ \\
Anastomosis in the lower & $59 \%$ & $55 \%$ & $0.644^{1}$ \\
two thirds of the rectum & $21 \%$ & $13 \%$ & $0.066^{1}$ \\
\hline
\end{tabular}

5.2 days (SD 2.1). In the case of an anastomotic leak, the most recent laboratory results before the diagnosis was made were assessed on mean 8.4 days (SD 4.5), the difference to the time the diagnosis was made was mean 0.5 days (SD 1.2).

Hyponatremia $(<136 \mathrm{mmol} / \mathrm{l})$ was present in $23 \%(n=19)$ of patients in the group with an anastomotic leak and in $15 \%$ $(n=69)$ of patients in the group without $(n=1,025, p<0.001)$. The serum sodium levels $(p<0.001)$ and leukocyte counts $(p<0.001)$ correlated with the presence of an anastomotic leak. In logistic regression analysis, both leukocyte counts $(p<0.001,95 \%$ CI 0.074 to 0.166$)$ and serum sodium levels $(p=0.001,95 \%$ CI 0.154 to -0.037$)$ remained independent significant markers of an anastomotic leak.

The significant differences of the mean serum sodium level and of mean leukocytes in the group of patients with an anastomotic leak and the group without are shown in Fig. 2 and in Fig. 3. Leukocytosis was present in 12 of the 19 patients with hyponatremia and an anastomotic leak. The sensitivities, specificities, positive predictive values, and the negative

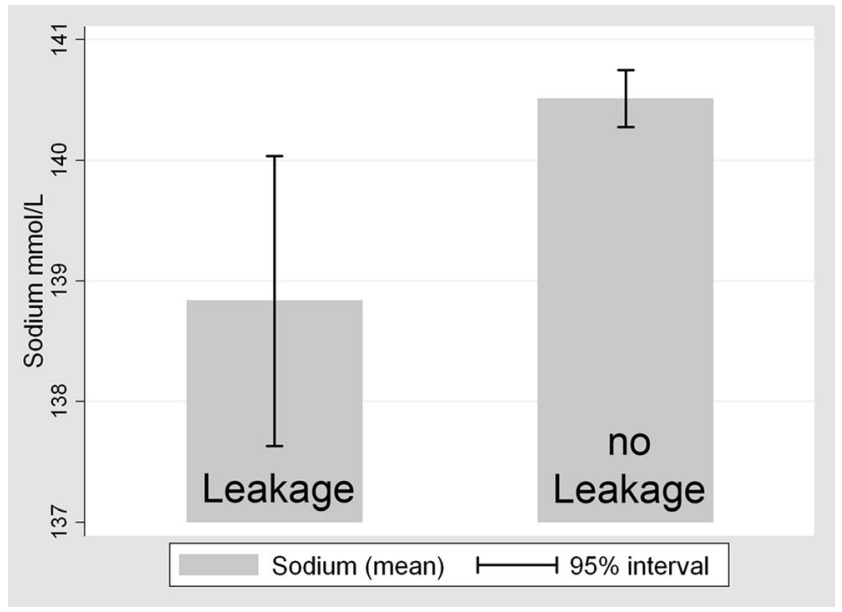

Fig. 2 Mean values of serum sodium levels in the presence and in the absence of an anastomotic leak after colorectal surgery (difference significant in the Wilcoxon rank sum test, $p<0.001$ ) predictive values of hyponatremia, leukocytosis, and the possible combinations are shown in Table 2. A receiver operation characteristic analysis with leakage as the reference variable and the sodium level as the classification variable showed a receiver operation characteristic area of 0.5016 on day 4 , 0.5337 on day $5,0.4050$ on day 6 , and 0.1176 on day 7 postoperative. The results of the sensitivity, specificity, and negative and positive predictive values of hyponatremia with regard to the different days postoperative are shown in Table 4.

Preoperative sodium level dropped by mean $1.3 \mathrm{mmol} / \mathrm{l}$ in the group of patients who developed an anastomotic leak but raised by mean $0.8 \mathrm{mmol} / \mathrm{l}$ in the group of patients who did not develop an anastomotic leak. The drop of preoperative sodium level was significantly associated with the development of anastomotic leak $(p<0.001)$. The results of a receiver operating characteristic analysis of the drop of preoperative sodium level with regard to the development of an anastomotic leak are shown in Table 3.

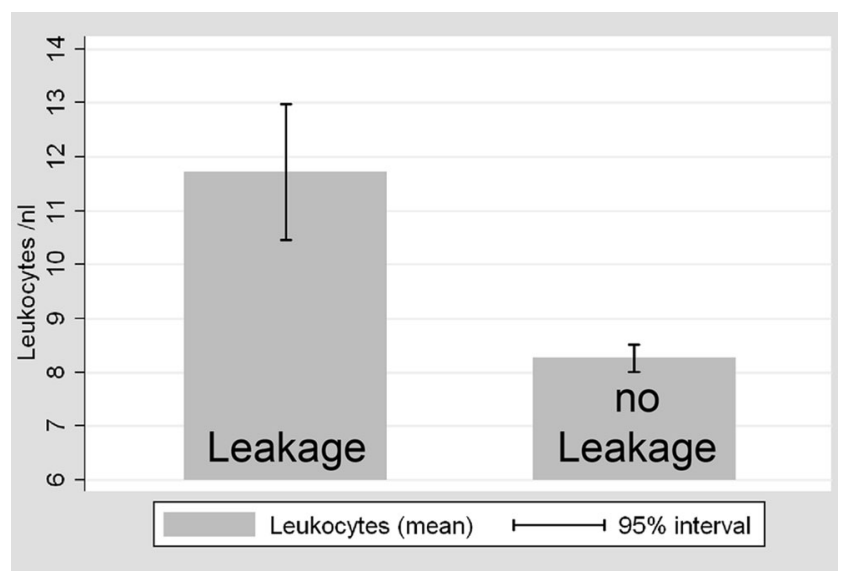

Fig. 3 Mean values of leukocytes in the presence and in the absence of an anastomotic leak after colorectal surgery (difference significant in the Wilcoxon rank sum test, $p<0.001$ ) 
Table 2 Sensitivities, specificities, and positive and negative predictive values of hyponatremia, leukocytosis, and the possible combinations

\begin{tabular}{lllll}
\hline & Sensitivity & Specificity & Positive predictive value & Negative predictive value \\
\hline Hyponatremia $(95 \% \mathrm{CI})$ & $24 \%(15-35)$ & $93 \%(91-95)$ & $22 \%(14-32)$ & $94 \%(92-95)$ \\
Leukocytosis $(95 \% \mathrm{CI})$ & $58 \%(47-69)$ & $79 \%(77-82)$ & $18 \%(14-24)$ & $96 \%(94-97)$ \\
Hyponatremia an Leukocytosis $(95 \% \mathrm{CI})$ & $14 \%(7-23)$ & $98 \%(96-98)$ & $31 \%(17-49)$ & $94 \%(92-95)$ \\
Hyponatremia or Leukocytosis $(95 \% \mathrm{CI})$ & $68 \%(56-78)$ & $75 \%(72-77)$ & $18 \%(14-23)$ & $97 \%(95-98)$ \\
\hline
\end{tabular}

CI confidence interval

The interval between surgery and diagnosis of an anastomotic leak was significantly longer in the subgroup of patients with hyponatremia (mean 12.4 days) compared to the group without (mean 7.4 days) ( $p=$ 0.003). Especially in the subgroup of patients with anastomotic leaks of anastomoses in the lower two thirds of the rectum with defunctioning ileostomies, the interval between surgery and diagnosis of an anastomotic leak was significantly longer in those with hyponatremia (mean 12.6 days) compared to those without (mean 5.7 days) $(p=0.009)$. In the absence of a defunctioning ileostomy, there was no significant difference in the intervals between surgery and diagnosis of an anastomotic leak between patients with hyponatremia (mean 9.9 days) compared to those without (mean 7.5 days $)(p=0.176)$.

\section{Discussion}

We aimed to define the significance of hyponatremia as a marker of anastomotic leaks after colorectal surgery. The most recent serum sodium levels and leukocyte counts measured before an anastomotic leak was diagnosed, were assessed, and were compared to the values in the absence of an anastomotic leak. Hyponatremia turned out to be a specific marker of an anastomotic leak after colorectal surgery.
Established inflammation markers such as elevated Creactive protein (CRP) and elevated leukocytes can be used for detection of anastomotic leaks after colorectal surgery [13]. A recent meta-analysis showed that a CRP below $135 \mathrm{mg} / \mathrm{l}$ on postoperative day 4 has a high negative predictive value ( $89 \%$ ) for infectious complications after colorectal surgery [14]. However, possibly due to expense, CRP is often not measured in the daily routine, while the serum sodium level is. Thus, the hypothesis that hyponatremia is a marker of anastomotic leakage after colorectal surgery could be of clinical importance.

Due to low sensitivity ( $23 \%$ ), the absence of hyponatremia cannot be used to exclude an anastomotic leak after colorectal surgery. But, as the specificity of hyponatremia for an anastomotic leak is as high as $93 \%$, the presence of hyponatremia should raise suspicion for an anastomotic leak after colorectal surgery.

In the case of suspicion for an anastomotic leak after colorectal surgery, a CT scan with contrast dye remains the preferred diagnostic modality to confirm the diagnosis [11]. However, routine CT scans for postoperative evaluation of anastomoses in colorectal surgery are not recommended. Furthermore, the results of CT studies performed to exclude anastomotic leaks after colorectal surgery are not always explicit [15], and false negative results can lead to a potentially disastrous delay in diagnosis and treatment [16]. Thus, it remains an

Table 3 Receiver operating characteristic analysis of the drop of preoperative sodium level with regard to the development of an anastomotic leak

\begin{tabular}{llllll}
\hline Cut point (drop of sodium level) & Sensitivity & Specificity & Correctly classified & Positive likelihood ratio & Negative likelihood ratio \\
\hline$\geq 0 \mathrm{mmol} / 1$ & $71 \%$ & $50 \%$ & $52 \%$ & 1.4 & 0.6 \\
$\geq 2 \mathrm{mmol} / 1$ & $54 \%$ & $70 \%$ & $69 \%$ & 1.8 & 0.7 \\
$\geq 4 \mathrm{mmol} / 1$ & $35 \%$ & $88 \%$ & $84 \%$ & 2.8 & 0.7 \\
$\geq 6 \mathrm{mmol} / 1$ & $17 \%$ & $95 \%$ & $89 \%$ & 3.4 & 0.9 \\
$\geq 8 \mathrm{mmol} / 1$ & $8 \%$ & $99 \%$ & $92 \%$ & 5.4 & 0.9 \\
$\geq 10 \mathrm{mmol} / 1$ & $5 \%$ & $99 \%$ & $92 \%$ & 7.2 & 1.0 \\
\hline
\end{tabular}

ROC area 0.64 
Table 4 Sensitivity, specificity, and negative and positive predictive values of hyponatremia with regard to different days postoperative (analysis limited to days 4-7 postoperative due to missing laboratory values in the case of uneventful postoperative course)

\begin{tabular}{lllll}
\hline Day postoperative & Sensitivity & Specificity & Negative predictive value & Positive predictive value \\
\hline Day 4 $(95 \% \mathrm{CI})$ & $10 \%(2-45)$ & $94 \%(90-97)$ & $96 \%(92-98)$ & $7 \%(1-34)$ \\
Day 5 $(95 \% \mathrm{CI})$ & $17 \%(3-48)$ & $90 \%(87-93)$ & $97 \%(95-99)$ & $5 \%(1-17)$ \\
Day 6 $(95 \% \mathrm{CI})$ & $13 \%(2-40)$ & $94 \%(89-97)$ & $92 \%(87-96)$ & $15 \%(2-46)$ \\
Day 7 $(95 \% \mathrm{CI})$ & $0 \%(0-83)$ & $94 \%(84-99)$ & $98 \%(89-100)$ & $0 \%(0-70)$ \\
\hline
\end{tabular}

CI confidence interval

important challenge to consistently and expeditiously determine whether and when anastomotic leakage after colorectal surgery should be suspected.

What is the pathophysiology of postoperative hyponatremia? Different non-osmotic stimuli such as pain, stress, nausea, emesis, and/or intravascular volume depletion result in increased secretion of vasopressin and consecutive water retention after surgical interventions [3]. These stimuli are much stronger in anastomotic leak with peritonitis and beginning sepsis syndrome resulting in pain, stress, release of inflammation mediators as interleukin 6 [4], and volume depletion due to loss of water into the third space and diarrhea [3]. The surface of the peritoneum is $1.3-1.4 \mathrm{~m}^{2}$ [17]. As the peritoneal cells have microvilli, the functional surface is even larger [18]. These pathophysiological mechanisms with high volume loss into the peritoneal cavity have been compared to severe burns with a high percentage of total body surface involved [19]. The water and electrolyte imbalance resulting from peritonitis can be further disturbed by the impaired renal function associated with the beginning sepsis syndrome [20] and by diuretics administration [3].

It could be argued that an anastomotic leakage provokes electrolyte imbalances, and therefore, it could be difficult to differentiate between cause and consequence. First, no significant difference in the prevalence of preoperative hyponatremia could be detected in the group of patients who developed an anastomotic leak compared with those who did not. Second, the drop of sodium level was a significant marker of the presence of an anastomotic leak. Thus, it is more likely that hyponatremia is the consequence of an anastomotic leak and that it is not a risk factor for the development of an anastomotic leak.

The interval between surgery and the day an anastomotic leak was diagnosed was significantly longer in the subgroup with hyponatremia, compared to the group without. This could indicate a bias. However, the most probable explanation for this finding is that a delay in diagnosing the anastomotic leak leads to a longer impact of the abovementioned factors leading to electrolyte disturbances. This finding is even pronounced in patients with anastomoses in the lower two thirds of the rectum with defunctioning ileostomies that ameliorate the clinical consequences of an anastomotic leak [21]. Thus, the diagnosis of an anastomotic leak might be delayed, as patients might suffer from a silent leak until the diagnosis is made. This is underlined by missing significant difference in the interval between surgery and the day an anastomotic leak was diagnosed in patients without a defunctioning ileostomy.

To clarify this, a receiver operation characteristic analysis with leakage as the reference variable and the sodium level as the classification variable was done; it showed the highest area under the curve on day 5 postoperative suggesting this day to be the best for diagnosis/prediciting anastomotic leakage by hyponatremia. The corresponding sensitivity, specificity, and negative and positive predictive values are shown in Table 4. However, as the sodium level was rarely measured after day 7 in patients with an uneventful postoperative course, this analysis was limited from day 4 to day 7 , and thus, the conclusions drawn are of limited significance. Indeed, a prospective trial with measurement of sodium on predefined days after colorectal surgery is needed to answer this question.

\section{Conclusion}

Hyponatremia seems to be a significant and clinically relevant marker of anastomotic leakage after colorectal surgery. The presence of hyponatremia should raise suspicion of an anastomotic leak after colorectal surgery, especially in patients with delayed recovery in the late postoperative course. If hyponatremia and leukocytosis are present after colorectal surgery, a CT scan with rectal contrast dye is recommended to exclude an anastomotic leak. However, further prospective trials are needed to confirm these results.

Conflicts of interest None 


\section{References}

1. Thompson C, Hoorn EJ (2012) Hyponatraemia: an overview of frequency, clinical presentation and complications. Best Pract Res Clin Endocrinol Metab 26(Suppl 1):S1-S6. doi:10.1016/S1521690X(12)00019-X

2. Leung AA, McAlister FA, Rogers SO Jr, Pazo V, Wright A, Bates DW (2012) Preoperative hyponatremia and perioperative complications. Arch Intern Med 172(19):1474-1481. doi:10.1001/ archinternmed.2012.3992

3. Thomas L (2008) Wasserbalance und Flüssigkeitsräume. In: Thomas L (ed) Labor und Diagnose, vol 1. TH-Books Verlagsgesellschaft mbH, Frankfurt/Main, pp 424-433

4. Swart RM, Hoorn EJ, Betjes MG, Zietse R (2011) Hyponatremia and inflammation: the emerging role of interleukin-6 in osmoregulation. Nephron Physiol 118(2):45-51. doi:10.1159/000322238

5. Sharshar T, Blanchard A, Paillard M, Raphael JC, Gajdos P, Annane D (2003) Circulating vasopressin levels in septic shock. Crit Care Med 31(6):1752-1758. doi:10.1097/01.CCM. $0000063046.82359 .4 \mathrm{~A}$

6. Nair V, Niederman MS, Masani N, Fishbane S (2007) Hyponatremia in community-acquired pneumonia. Am J Nephrol 27(2):184-190. doi: $10.1159 / 000100866$

7. Sigal SH (2012) Hyponatremia in cirrhosis. J Hosp Med Off Publ Soc Hosp Med 7(Suppl 4):S14-S17. doi:10.1002/jhm.1915

8. Clowes GH Jr, Vucinic M, Weidner MG (1966) Circulatory and metabolic alterations associated with survival or death in peritonitis: clinical analysis of 25 cases. Ann Surg 163(6):866-885

9. Käser SA, Furler R, Evequoz DC, Maurer CA (2013) Hyponatremia is a specific marker of perforation in sigmoid diverticulitis or appendicitis in patients older than 50 years. Gastroenterol Res Pract 2013: 462891. doi:10.1155/2013/462891

10. Adrogue HJ, Madias NE (2000) Hyponatremia. N Engl J Med 342(21): 1581-1589. doi:10.1056/NEJM200005253422107

11. Hyman N, Manchester TL, Osler T, Burns B, Cataldo PA (2007) Anastomotic leaks after intestinal anastomosis: it's later than you think. Ann Surg 245(2):254-258. doi:10.1097/01.sla.0000225083. 27182.85
12. Rahbari NN, Weitz J, Hohenberger W, Heald RJ, Moran B, Ulrich A, Holm T, Wong WD, Tiret E, Moriya Y, Laurberg S, den Dulk M, van de Velde C, Buchler MW (2010) Definition and grading of anastomotic leakage following anterior resection of the rectum: a proposal by the International Study Group of Rectal Cancer. Surgery 147(3): 339-351. doi:10.1016/j.surg.2009.10.012

13. Almeida AB, Faria G, Moreira H, Pinto-de-Sousa J, Correia-da-Silva P, Maia JC (2012) Elevated serum C-reactive protein as a predictive factor for anastomotic leakage in colorectal surgery. Int J Surg 10(2): 87-91. doi:10.1016/j.ijsu.2011.12.006

14. Warschkow R, Beutner U, Steffen T, Muller SA, Schmied BM, Guller U, Tarantino I (2012) Safe and early discharge after colorectal surgery due to C-reactive protein: a diagnostic meta-analysis of 1832 patients. Ann Surg 256(2):245-250. doi:10.1097/SLA. 0b013e31825b60f0

15. Khoury W, Ben-Yehuda A, Ben-Haim M, Klausner JM, Szold O (2009) Abdominal computed tomography for diagnosing postoperative lower gastrointestinal tract leaks. J Gastrointest Surg Off J Soc Surg Aliment Tract 13(8):1454-1458. doi:10.1007/ s11605-009-0925-4

16. Doeksen A, Tanis PJ, Vrouenraets BC, van Lanschot JJ, van Tets WF (2007) Factors determining delay in relaparotomy for anastomotic leakage after colorectal resection. World J Gastroenterol WJG 13(27): 3721-3725

17. Mörl F, Dittmer H (1984) Peritonitis. In: L. D (ed) Klinische Gastroenterologie, vol 1. Thieme, Stuttgart, pp 787-792

18. Strobel M, Beger H (1987) Struktur des Peritoneums. In: BHK E (ed) Akutes Abdomen. Thieme, Stuttgart, New York

19. Webb WR (1966) Comment on: circulatory and metabolic alterations associated with survival or death in peritonitis: clinical analysis of 25 cases. Ann Surg 163:885

20. Rangel-Frausto MS, Pittet D, Costigan M, Hwang T, Davis CS, Wenzel RP (1995) The natural history of the systemic inflammatory response syndrome (SIRS). A prospective study. JAMA J Am Med Assoc 273(2):117-123

21. Huser N, Michalski CW, Erkan M, Schuster T, Rosenberg R, Kleeff J, Friess H (2008) Systematic review and meta-analysis of the role of defunctioning stoma in low rectal cancer surgery. Ann Surg 248(1): 52-60. doi:10.1097/SLA.0b013e318176bf65 